Marta Maskey-Warzęchowska', Marta Dąbrowska', Rafał Krenke', Anna Domeracka-Kołodziej', Małgorzata Żukowska ${ }^{3}$, Ryszarda Chazan'

'Department of Internal Medicine, Pneumonology and Allergology, Medical University of Warsaw

2Department of Otolaryngology, Medical University of Warsaw, Poland

${ }^{3}$ Department of Radiology, Medical University of Warsaw, Poland

\title{
Left brachiocephalic vein stenosis and infectious aortitis: two unusual causes of Ortner's syndrome
}

The authors declare no financial disclosure

\begin{abstract}
Ortner's syndrome (also known as cardiovocal syndrome) is defined as hoarseness due to compression of the left recurrent laryngeal nerve by an enlarged left atrium or enlarged thoracic vessels. We describe two cases of Ortner's syndrome with an unusual underlying vascular pathology. In the first patient, Ortner's syndrome was a consequence of left brachiocephalic vein stenosis resulting in collateral circulation filling the aorto-pulmonary window. The second patient developed a thoracic aortic aneurysm due infectious aortitis. Both patients required careful scrutiny in differential diagnosis because of their complex past medical history and concomitant diseases.
\end{abstract}

Key words: hoarseness, vocal cord paresis, left recurrent laryngeal nerve palsy, Ortner's syndrome, cardiovocal syndrome

Pneumonol Alergol Pol 2015; 83: 457-461

\section{Introduction}

In 1897, Norbert Ortner described a clinical entity characterized by hoarseness due to left recurrent laryngeal nerve (LRLN) palsy caused by the compression of the left recurrent laryngeal nerve by an enlarged left atrium in patients with mitral stenosis [1]. In 1958, the term 'cardiovocal syndrome' was introduced to describe LRLN palsy not only in the course of mitral stenosis, but also in other cardiovascular disorders including aneurysms of the aorta or the pulmonary artery, aneurysms of the left ventricle, congenital heart defects, pulmonary hypertension and tortuosity of the great vessels. Cardiovocal syndrome has also been described in patients after cardiothoracic surgical interventions and atrial fibrillation ablation procedures [2].

The exact cause of the LRLN palsy in Ortner's syndrome is still being discussed. Currently, the strongest hypothesis is that it is a result of LRLN compression in the aorto-pulmonary window (by the dilated aorta, pulmonary artery or any other pathologic vascular structure in this anatomical region) [2].

We report two cases with Ortner's syndrome with unusual underlying conditions.

\section{Case 1}

An 80-year-old woman presented to the pneumonology department with a 4-week history of exertional dyspnea and hoarseness. Two weeks prior to admission, she was diagnosed with deep venous thrombosis of the left lower limb and had been treated with enoxaparin. Her medical history also included right sided mastectomy followed by radiation and chemotherapy due to breast cancer nine years ago and she was currently receiving capecitabine because of disease progression (metastases to the liver).

Address for correspondence: Marta Maskey-Warzęchowska, Department of Internal Medicine, Pneumonology and Allergology, Medical University of Warsaw

ul. Banacha 1a, 02-097 Warsaw, Poland, e-mail: mmaskey@op.pl.

DOI: 10.5603/PiAP.2015.0075

Received: 17.09 .2015

Copyright (C) 2015 PTChP

ISSN 0867-7077 


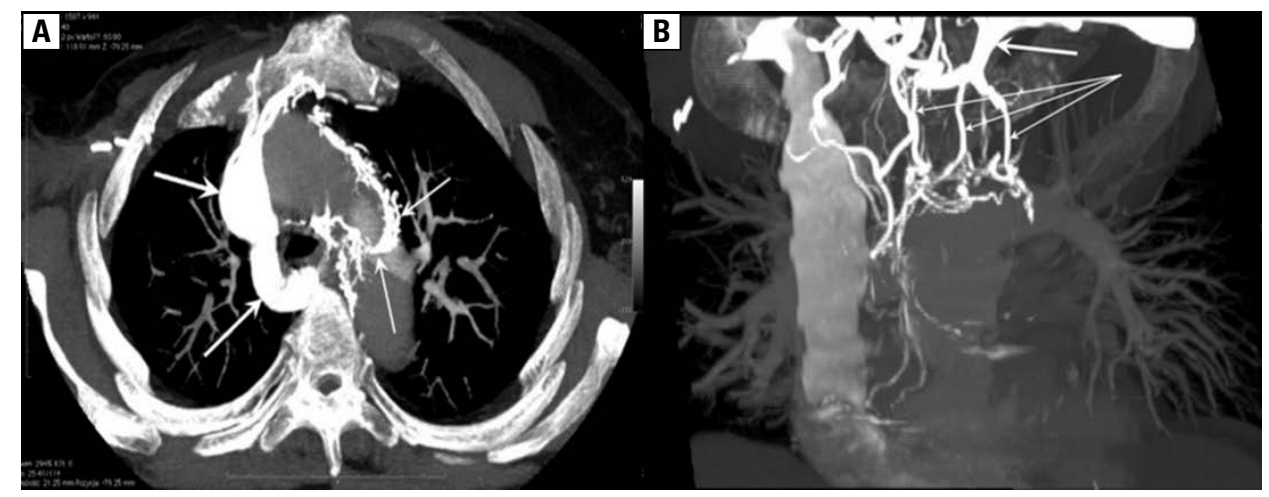

Figure 1. A. Axial computed tomography scan of the upper thorax. Systemic veins are shown in white; enlarged collateral veins localized in the aorto-pulmonary window (small arrows), enlarged azygos vein (medium sized arrow) and superior vena cava (big arrow). Ascending and descending aorta can be seen in dark grey; B. Computed tomography reconstruction of the left brachiocephalic vein (big arrow) and enlarged collateral vessels (small arrows) surrounding the aortic arch (removed from the image) filling the aorto-pulmonary window and bring blood to superior vena cava (coronal oblique view). Pulmonary arteries are seen in dark grey

Physical examination revealed edema of the left calf. No abnormality on examination of the chest was found. With the exception of an elevated d-dimer level ( $848 \mathrm{ng} / \mathrm{mL}$, normal range 0-500 $\mathrm{ng} / \mathrm{mL}$ ), the results of her laboratory studies were irrelevant. The chest radiograph was normal. Spiral multidetector computed tomography (CT) of the chest did not confirm the initial diagnosis of pulmonary embolism.

Left vocal cord palsy was revealed on laryngological examination and this was diagnosed as the cause of hoarseness. However, no obvious causes for vocal cord palsy were found. There were no pathological masses within the lungs or enlarged mediastinal lymph nodes, which could be responsible for the left laryngeal nerve paralysis. Nevertheless, multiplanar CT reconstructions of the intrathoracic vessels revealed a $90 \%$ stenosis in the proximal fragment of the left brachiocephalic vein with collateral circulation surrounding the aortic arch and filling the space of the aorto-pulmonary window (Fig. 1). The stenosis was caused by the tight space between the posterior wall of the manubrium sterni and the arch of the aorta. A dilatation of the pulmonary trunk to $36 \mathrm{~mm}$ was also noted.

As other investigations (CT of the neck, bronchoscopy) failed to identify any other cause of the left vocal cord palsy, the compression of the left recurrent laryngeal nerve by the collateral venous circulation and the dilated pulmonary trunk was assumed the most probable cause of hoarseness. This was consistent with diagnosis of cardiovocal syndrome (Ortner's syndrome). Anticoagulation therapy with enoxaparin was continued, no additional treatment was offered; the patient was discharged and observed on an ambulatory basis, the hoarseness and exertional dyspnea did not improve.

\section{Case 2}

A 53-year-old man who underwent endovascular graft stenting for a thoracic aortic aneurysm 2 weeks prior to admission presented to the pneumonology department due to hoarseness and hemoptysis which had started 2 months earlier and persisted after the endovascular procedure. Since 3 years, he had been treated with methylprednisolone $10 \mathrm{mg} /$ day and methotrexate $20 \mathrm{mg}$ once a week due to rheumatoid arthritis and currently had no complaints associated with the disease. The patient was in a good general condition, afebrile; physical examination revealed wheezing in the upper area of the left lung. Laryngological examination showed left vocal cord palsy.

The results of the laboratory studies showed a slightly elevated serum C-protein level $-20.0 \mathrm{mg} / \mathrm{L}$ (normal range $0.0-10.0 \mathrm{mg} / \mathrm{L}$ ); the white blood cell count was normal. The results of other laboratory studies were irrelevant. The chest radiograph showed a widening of the upper left mediastinum which corresponded with the aneurysm of the descending aorta seen on CT. The aneurysm extended to the left subclavian artery and filled the aorto-pulmonary window (Fig. 2).

Considering the absence of pathological masses in the chest CT and the location of the aneury$\mathrm{sm}$, cardiovocal syndrome was assumed the cause of hoarseness. However, because the CT showed an ill-defined parenchymal opacity adjacent to the aneurysm (Fig. 3A), fiberoptic bronchoscopy was performed. Culture of bronchial washing from the left apico-posterior segment $(\mathrm{LB} 1+2)$ was positi- 


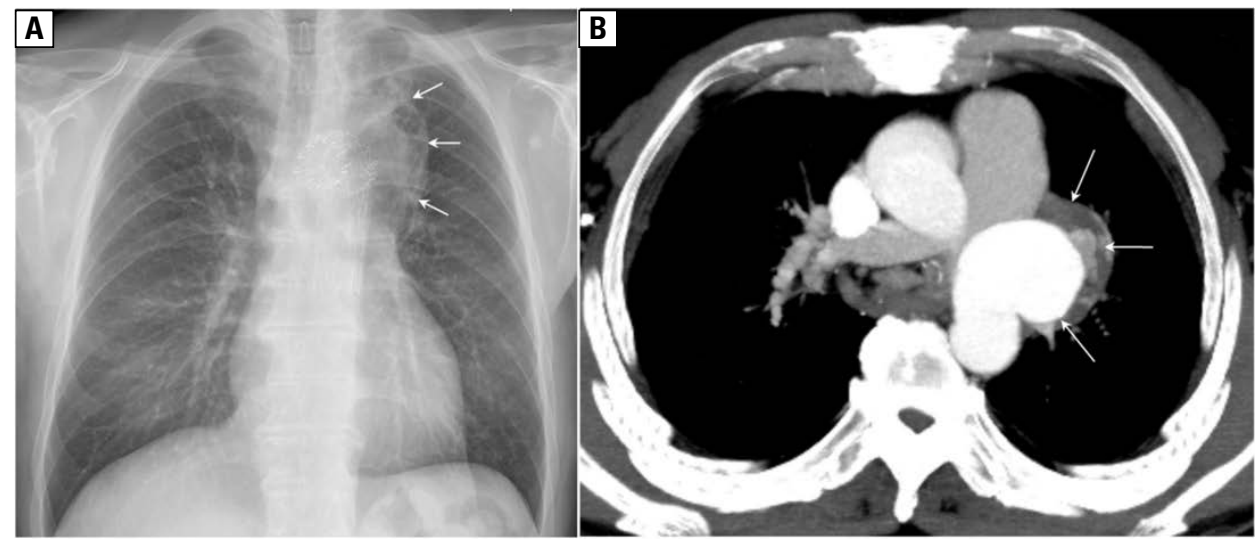

Figure 2. A. Antero-posterior chest radiograph showing an inhomogeneous opacity in the left upper area of the left lung. The lesion is adjacent to the left mediastinum and has a relatively well defined left margin (arrows). The endovascular stent is visible in the aortic arch and descending aorta; B. Axial computed tomography scan of the large vessels at the level of the aorto-pulmonary window. Arrows show the external margin of the aneurysm. Contrast medium is seen in the aneurysm (white colour), but also in the ascending aorta and superior vena cava. Pulmonary trunk and the right pulmonary artery are shown in grey

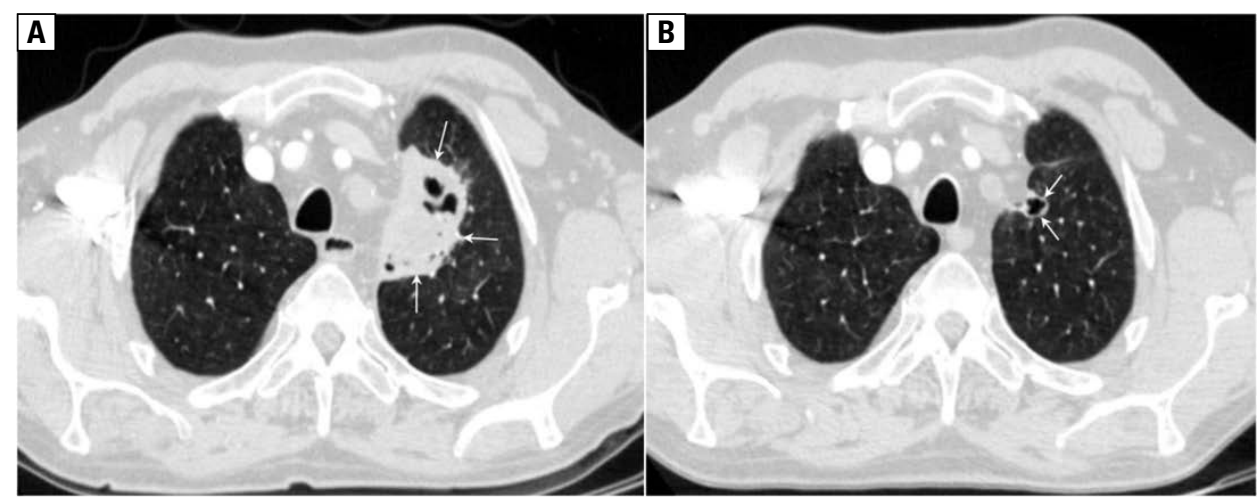

Figure 3. A. Axial computed tomography scan of the thorax at the level above the aortic arch (pulmonary window). A large, irregular consolidation with cavitations adjacent to the mediastinum is seen in the upper lobe of the left lung (arrows); B. An almost complete resolution of the consolidation in the upper lobe of the left lung (only a small cavitation is seen) after directed antibiotic therapy

ve for Salmonella enteritidis. Blood cultures were negative. Microbiological studies of the bronchial washing samples for Mycobacterium tuberculosis gave negative results. The patient received directed antimicrobial treatment (ceftriaxone) for 6 weeks with clinical improvement. Bronchial washing culture performed after the completion of antibiotic therapy gave negative results. In a follow up CT after 3 months, a regression of the above mentioned opacity was noted and the dimensions of the aneurysm were significantly reduced (Fig. 3B), the hoarseness, however, was still present.

\section{Discussion}

Initially, the term 'Ortner's syndrome' was used to describe hoarseness due to LRLN palsy secondary to LRLN compression by an enlarged left atrium in patients with mitral stenosis [1]. Over the years, the definition of Ortner's syndrome had evolved and it is currently applied to describe hoarseness associated with LRLN palsy in various pathologies of the large thoracic vessels and the heart, and thus the term 'cardiovocal syndrome' may seem more appropriate. In our two presented cases, Ortner's syndrome was associated with rare vascular diseases and both of them required scrutinous differential diagnosis because of their concomitant diseases.

In our first patient, Ortner's syndrome could be attributed to the stenosis of the left brachiocephalic vein (BCV) which triggered the development of an abundant collateral venous circulation. The collateral vessels entered the aorto-pulmonary window causing LRLN compression. To our knowledge, such a cause of cardiovocal syndrome had not been reported previously. CT imaging indicated the tight space between the manubrium of the sternum and the arch of the 
aorta as the cause of the left BCV compression and stenosis. The etiology of this anatomical alteration is unclear. The patient had no history of chest trauma or surgical procedures with thoracotomy. A congenital abnormality should also be taken into account, however, the patient's radiological history was too short to confirm a congenital rib cage deformation.

Other potential causes of BCV stenosis include obstruction by mediastinal tumors, complicated central vein catheterization and thrombosis [3, 4]. These conditions were excluded in our patient, as she had not received chemotherapy via a central venous catheter during the treatment of breast cancer and there was no evidence for venous thrombosis or pathological masses in the mediastinum on CT.

The dilated pulmonary trunk could also contribute to Ortner's syndrome. The cause of this dilatation is not clear; the normal appearance of the right ventricle with a normal tricuspid regurgitation pressure gradient on transthoracic echocardiography allowed to exclude significant pulmonary hypertension. As the appearance of the lungs in CT was normal, interstitial lung disease was not considered as a potential cause of pulmonary hypertension in our patient. The patient did not have any signs of autoimmune disease, however, serum autoantibody titers were not investigated. An idiopathic character of the dilatation cannot be excluded $[5,6]$.

Aortic aneurysm has been described as one of the most frequent causes of cardiovocal syndrome. However, in our second patient, the aneurysm of the descending aorta could be related to Salmonella enteritidis infection. Salmonella aortitis is a rare entity with a mortality rate as high as $28-41 \%$, mainly associated with delay in diagnosis resulting in subsequent aneurysm rupture or infection related complications [7-9]. Infectious aortitis may develop as a result of extension of the infection from an adjacent focus, direct infection of an injured intima or atherosclerotic plaque or may be caused by septic emboli in the vasa vasorum $[10,11]$. Due to a certain resemblance to fungal growth on gross appearance, aneurysms secondary to infectious aortitis are misleadingly referred to as mycotic aortic aneurysms (MAAs). MAAs are more frequent in elderly men, immunodeficiency is a significant risk factor [9, 12]. They account for $0.65-2.0 \%$ of all aortic aneurysms in the Western countries [9].

Hoarseness and thoracic or back pain in febrile patients with a Salmonella positive blood culture may suggest Salmonella aortitis and require immediate attention and investigation of the aorta $[7,10]$. The clinical paucity of Salmonella infection in our patient could have been a result of immunosuppressive therapy. Blood cultures were negative; we did, however, obtain a Salmonella positive culture of the bronchial washing samples taken from the left upper lobe, a region adjacent to the aneurysm, where inflammatory changes were seen on CT. The patient was successfully treated by endovascular repair and directed antibiotic therapy. Endovascular aortic repair (EVAR) together with antimicrobial therapy is the treatment of choice in MAAs [9, 12].

There were two other potential causes of aortitis leading to aneurysm formation in this patient. Aortitis is a rare vascular complication of rheumatoid arthritis (RA) [11], a disease for which the patient had been treated for three years. Gravallese et al. reported a series of $10 \mathrm{RA}$ patients who had features of aortitis on autopsy, 3 of these patients had a diagnosis of aortic aneurysm ( 2 thoracic, 1 abdominal) before death. In all 3 cases, the aneurysms had developed in regions affected by aortitis [13]. Taking into account the improvement after antibiotics without any modifications of immunosuppressive treatment in our patient, the probability of an aneurysm secondary to RA-related aortitis seems low.

Tuberculous aortitis should also have been taken into consideration, particularly in the presence of the abnormal appearance of the left upper lung in chest CT and in the light of chronic immunosuppression. This condition, however, is extremely rare with less than 50 cases described in the English literature from the introduction of antituberculous treatment till 1999 [14]. Tuberculous aneurysms are more likely to affect the descending aorta. The mechanisms leading to aortic wall infection and destruction in tuberculosis are similar to those in other bacterial aortitides, the probability of tuberculous aortitis seems to be higher in disseminated (blood-borne) tuberculosis. Hoarseness was not reported as one of the presenting symptoms in the analyzed cases [14]. In our patient, tuberculosis was excluded by negative microscopic evaluation and negative $M$. tuberculosis cultures from sputum and bronchial washing samples. This was further strengthened by the clinical and radiological improvement after non-antituberculous chemotherapy.

The treatment of cardiovocal syndrome is directed on the treatment of the underlying disease, and the outcome depends on the degree and duration of LRLN injury [2]. In Case 1 no causal treatment could be offered, in Case 2 hoarseness 
also persisted 3 months after the endovascular repair and antibiotic therapy, despite of the reduction of the aneurysm dimensions (the patient was lost to further follow-up). However, a report of complete hoarseness resolution 1 year after an endovascular stent implantation was published [15].

In conclusion, Ortner's syndrome (also known as cardiovocal syndrome) is a relatively rare entity characterized by hoarseness due to left recurrent laryngeal nerve palsy caused by its compression by the enlarged chambers of the heart or enlarged thoracic vessels. In patients with a complex past medical history, it requires a detailed diagnostic work-up and is often a diagnosis of exclusion. The hoarseness usually persists after treatment of the underlying disease, however, a complete resolution of Ortner's syndrome has been reported after endovascular repair and the reduction of LRLN compression.

\section{Conflict of interest}

The authors declare no conflict of interest

\section{References:}

1. Ortner N. Recurrenslahmung bei mitral stenoze. Wien Klin Wochenschr 1897; 10: 753-755.
2. Mulpuru SK, Vasavada BC, Punukollu GK, Patel AG. Cardiovocal syndrome: a systematic review. Heart, Lung and Circulation 2008; 17: 1-4.

3. Joffe HV, Goldhaber SZ. Upper extremity deep vein thrombosis. Circulation 2002; 106: 1874-1880.

4. Ko SF, Huang CC, Ng SH et al. Imaging of the brachiocephalic vein. AJR 2008; 191: 897-907.

5. Ring NJ, Marshall AJ. Idiopathic dilatation of the pulmonary artery. Brit J Radiol 2002; 75: 532-535.

6. Sengupta A, Dubey SP, Chaudhuri D et al. Ortner's syndrome revisited. J Laryng Otol 1998; 112: 377-379.

7. Ting ACW, Cheng SWK, Ho P, Poon JTC. Endovascular repair for multiple Salmonella mycotic anerysms of the thoracic aorta presenting with cardiovocal syndrome. Eur J Card Thorac Surg 2004; 26: 221-224.

8. Hsu RB, Lin FY. Infected aneurysm of the thoracic aorta. J Vasc Surg 2008; 47: 270-276.

9. Sörelius K, Mani K, Björk $\mathrm{M}$ et al for the European MAA collaborators. Endovascular treatment of mycotic aortic aneurysms. A European Multicenter Study. Circulation 2014; 130: 2136-2142.

10. Foote EA, Postier RG, Greenfield RA, Bronze MS. Infectious aortitis. Curr Treat Options Cardiovasc Med 2005; 7: 89-97.

11. Gornik HL, Creager MA. Aortitis. Circulation 2008; 117: 30393051.

12. Soravia-Dunand VA, Loo VG, Salit IE. Aortitis due to Salmonella; report of 10 cases and comprehensive review the literature. Clin Inf Dis 1999; 29: 862-868.

13. Gravallese EM, Corson JM, Coblyn JS, Pinkus GS, Weinblatt ME. Rheumatoid arthritis: a rarely recognized but clinically significant entity. Medicine 1989; 68: 95-106.

14. Long R, Guzman R, Greenberg H, Safneck J, Hershfeld E. Tuberculous mycotic aneurysm of the aorta. Review of published medical and surgical experience. Chest 1999; 115: 522-531.

15. Stoob K, Alkadhi H, Lachat M et al. Resolution of hoarseness after endovascular repair of thoracic aortic aneurysm: a case of Ortner's syndrome. Ann Otol Rhinol Laryngol 2004; 113: $43-45$. 